\title{
BMJ Postoperative atrial fibrillation predicts Open long-term survival after aortic-valve surgery but not after mitral-valve surgery: a retrospective study
}

\author{
Nicolas Girerd, ${ }^{1}$ Julien Magne,${ }^{2}$ Philippe Pibarot, ${ }^{1}$ Pierre Voisine, ${ }^{3}$ \\ François Dagenais, ${ }^{3}$ Patrick Mathieu ${ }^{3}$
}

To cite: Girerd N, Magne J, Pibarot P, et al. Postoperative atrial fibrillation predicts long-term survival after aortic-valve surgery but not after mitral-valve surgery: a retrospective study. BMJ Open 2011;1:e00385.

doi:10.1136/

bmjopen-2011-000385

- Prepublication history for this paper is available online. To view these files please visit the journal online (http:// bmjopen.bmj.com).

Received 7 September 2011 Accepted 9 September 2011

This final article is available for use under the terms of the Creative Commons Attribution Non-Commercial 2.0 Licence; see http://bmjopen.bmj.com

${ }^{1}$ Department of Medicine, Laval University, Quebec, Canada

2Department of Medicine, University of Liège, Liège, Belgium

${ }^{3}$ Department of Surgery, Laval University, Quebec, Canada

Correspondence to Dr Patrick Mathieu; patrick.mathieu@chg.ulaval.ca

\section{ABSTRACT}

Background: Postoperative atrial fibrillation (POAF) has been reported to be associated with reduced longterm survival after isolated coronary artery bypass grafting surgery. The objective of this study was to determine the impact of POAF on long-term survival after valvular surgery.

Methods: The authors retrospectively analysed the preoperative and operative data of 2986 consecutive patients with no preoperative history of atrial fibrillation undergoing first valvular surgery (aorticvalve replacement (AVR), mitral valve replacement or mitral valve repair (MVR/MVRp) with or without coronary artery bypass grafting surgery) in their institution between 1995 and 2008 (median follow-up 5.31 years, range $0.1-15.0$ ). The authors investigated the impact of POAF on survival using multivariable Cox regression.

Results: Patients with POAF were older, and were more likely to have hypertension or renal failure when compared with patients without POAF. The 12-year survival in patients with POAF was $45.7 \pm 2.8 \%$ versus $61.4 \pm 2.1 \%$ in patients without POAF $(p<0.001)$. On a multivariable analysis, when adjusting for age and other potential confounding factors, POAF tended to be associated with lower long-term survival (HR for allcause death $(\mathrm{HR})=1.17,95 \% \mathrm{Cl} 1.00$ to $1.38, \mathrm{p}=0.051$ ). The authors also analysed this association separately in patients with AVR and those with MVR/MVRp. In the multivariable analysis, POAF was a significant predictor of higher long-term mortality in patients with AVR ( $\mathrm{HR}=1.22, \mathrm{Cl} 1.02$ to $1.45, \mathrm{p}=0.03)$ but not in patients with MVR/MVRp ( $\mathrm{HR}=0.87, \mathrm{Cl} 0.58$ to $1.29, \mathrm{p}=0.48$ ).

Conclusions: POAF is significantly associated with long-term mortality following AVR but not after MVR/ MVRp. The underlying factors involved in the pathogenesis of POAF after MVR/MVRp may partially account for the lack of association between POAF and survival in these patients.

\section{INTRODUCTION}

Postoperative atrial fibrillation (POAF) is the most frequent complication following cardiac surgery. Indeed, POAF occurs in

\section{ARTICLE SUMMARY}

Article focus

n To investigate if postoperative atrial fibrillation (POAF) may affect long-term survival following heart-valve surgery.

\section{Key messages}

- POAF is significantly associated with long-term mortality following aortic-valve replacement (AVR).

- POAF is not associated with long-term mortality following mitral valve replacement/repair.

Strengths and limitations of this study

- This study indicates that POAF is a significant predictor of long-term survival after AVR.

- Hence, additional specific intervention, possibly a closer follow-up, should be considered in these patients.

- This is an observational study, hence causality between POAF and long-term survival following AVR cannot be ascertained.

$20-50 \%$ of patients, depending on the type of surgical procedure. ${ }^{1}$ POAF is associated with higher morbidity, prolonged hospitalisation and increased hospital costs following cardiac surgery. ${ }^{1}$ However, conflicting results have been reported regarding the impact of POAF on early postoperative mortality. ${ }^{2}{ }^{3}$ Moreover, recent studies have shown that $\mathrm{POAF}$ is associated with reduced long-term survival after isolated coronary artery bypass grafting surgery $(\mathrm{CABG})$ in several large observational studies. ${ }^{4-8}$ Nonetheless, in the context of valvular surgery, few studies have examined the impact of POAF on long-term survival, and these studies have yielded conflicting results. ${ }^{6}{ }^{9}$ Mariscalco and Engstrom $^{6}$ reported that POAF is associated with lower survival after CABG but not after valvular surgery, whereas Filardo $e t a l^{9}$ found that POAF is significantly associated with increased long-term risk of mortality after aortic-valve replacement (AVR). 
The objective of this study was thus to examine the impact of POAF on long-term mortality after valvular surgery in a large cohort of patients. Further, we determined whether the impact of POAF on survival following valvular surgery was influenced by the treated valve (aortic or mitral) or the presence of concomitant coronary artery disease $(\mathrm{CAD})$ requiring $\mathrm{CABG}$.

\section{METHODS}

\section{Study population}

Data on 2986 patients with no preoperative history of paroxysmal or chronic atrial fibrillation (AF) operated on for a first valvular surgery at the Quebec Heart \& Lung Institute between 1995 and 2008 were prospectively collected in a computerised database and then retrospectively analysed. Patients with AVR, mitral valve replacement (MVR) and mitral valve repair (MVRp) were included. Previous or associated CABG was not considered as an exclusion criterion. Patients with at least one of the following criteria were excluded: (1) concomitant cardiac or vascular procedure (including tricuspid and pulmonary-valve surgery), (2) double-valve procedure (concomitant mitral and aortic-valve surgery), (3) previous valvular surgery and (4) short-term mortality (defined as death from any cause within 30 days after operation if the patient was discharged from hospital or within any interval if the patient was not discharged). The study was approved by the Institutional Review Board of Quebec Heart \& Lung Institute.

\section{Detection and treatment of $\mathrm{AF}$}

Detailed information regarding the detection and treatment of POAF in our institution was previously reported. ${ }^{1011}$ Briefly, POAF was defined as any sustained episode recorded during the postoperative hospital stay and requiring medical and/or electrical cardioversion. No systematic prophylactic measures were used to prevent the development of POAF. However, as a general rule, unless contraindicated, $\beta$-blocker medication was introduced (or reintroduced) in all patients within the first $24 \mathrm{~h}$ after surgery. A constant ambulatory electrocardiographic (ECG) monitoring was performed in all patients during the first $48 \mathrm{~h}$ after surgery. Thereafter, a standard daily 12-lead ECG was recorded. In every patient suspected of an arrhythmic event, a standard 12lead ECG was performed, and the patient was monitored with continuous ambulatory ECG until $48 \mathrm{~h}$ after the resolution of the arrhythmia. All patients with POAF were treated with intravenous amiodarone as a first-line therapy. When needed, electrical cardioversion was performed.

\section{Outcome}

Long-term survival data were obtained from the death certificates of the Registry Office of the Quebec government. All-cause mortality was analysed. Survival follow-up was closed on 31 December 2009.
Statistical analysis

Continuous variables were expressed as mean or median $\pm \mathrm{SD}$ and were compared using $\mathrm{t}$ tests for independent samples. Differences in proportion were compared using a $\chi^{2}$ test. We used the Kaplan-Meier method to estimate survivals. Differences between survival curves were analysed using the logrank test. The association between POAF and risk of all-cause was examined using individual and multivariable Cox proportional hazard models. In the multivariable model, we adjusted for the following potential confounders: age, gender, diabetes, hypertension, renal failure, chronic obstructive pulmonary disease, previous myocardial infarction, previous stroke, current smoking status and left ventricular systolic dysfunction defined as left-ventricular ejection fraction $<50 \%$. The choice of the covariates was based on current available evidence in the field of cardiac surgery rather than data-driven. Multiplicative interaction with POAF was tested for the following predefined variables: type of surgical procedure, type of underlying valvular dysfunction (regurgitation vs stenosis), severity of $\mathrm{CAD}$ and mechanical-valve implantation. A probability value $<0.05$ was considered significant. All statistical analyses were performed with SPSS V.15.

\section{RESULTS}

Baseline characteristics of patients with and without POAF Patients with POAF were older $(p<0.001)$ and had a higher prevalence of hypertension $(\mathrm{p}=0.03)$ and renal failure $(p=0.001)$ (table 1$)$. Patients receiving mechanical valves or current smokers were less likely to have POAF ( $\mathrm{p}=0.004$ and $<0.001$ respectively).

\section{Impact of POAF on long-term survival in the whole cohort}

The median follow-up was 5.31 years (range: $0.1-15.0$ years). Overall, 705 patients $(23.6 \%)$ died. The 6- and 12-year survival in patients with and without POAF were $78.1 \pm 1.3 \%$ and $45.7 \pm 2.8 \%$ versus $83.6 \pm 1.0 \%$ and $61.4 \pm 2.1 \%$, respectively $(\mathrm{p}<0.001$, figure 1$)$.

On multivariable analysis, when adjusting for age and other potential confounding factors, POAF tended to be associated with long-term survival $(\mathrm{HR}=1.17,95 \% \mathrm{CI}$ 1.00 to $1.38, \mathrm{p}=0.051$ ) (table 2).

There was no significant interaction with concomitant CABG, three-vessel disease or mechanical-valve implantation (all $\mathrm{p}>0.20$ ). Of note, the interaction with the type of valvular procedure (aortic-valve surgery versus mitral-valve surgery) was of borderline statistical significance $(p=0.09)$. Consequently, we also report results in subsets of patients with AVR or with MVR-MVRp.

Baseline characteristics of patients with and without POAF in patients with aortic-valve surgery and in patients with mitral-valve surgery

Patients with POAF were older and had a higher prevalence of renal failure regardless of the surgical procedure (table 1). Patients with hypertension and patients implanted with mechanical valves were more likely to 


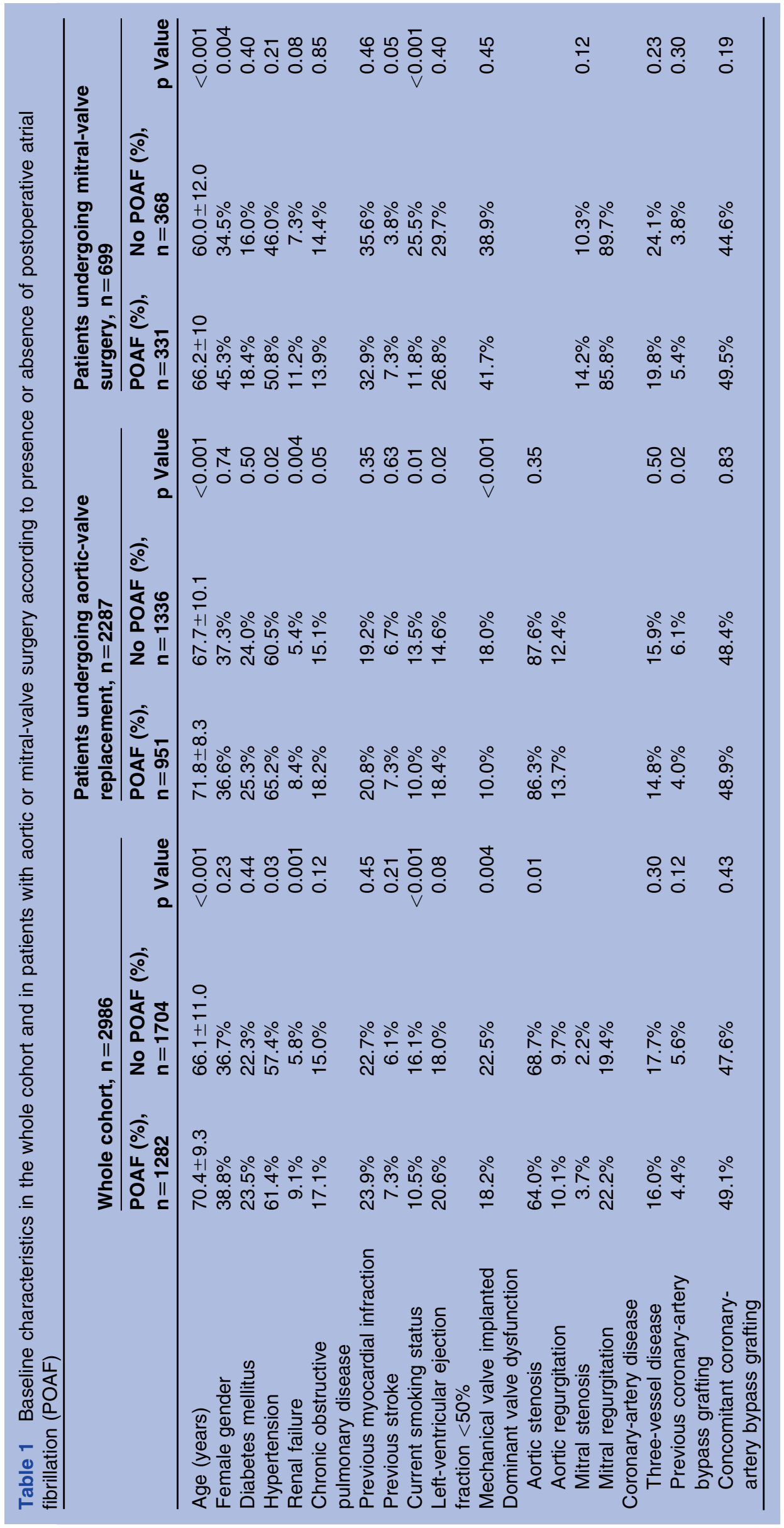


have POAF after aortic-valve surgery $(\mathrm{p}=0.02$ and $\mathrm{p}<0.001$ respectively), but not after mitral-valve surgery $(\mathrm{p}=0.21$ and $\mathrm{p}=0.45$ respectively). Current smokers were, in both groups, less likely to have POAF $(\mathrm{p}<0.001)$.

Impact of POAF on long-term survival in patients with aorticvalve surgery and in patients with mitral-valve surgery

On individual analysis, POAF was associated with reduced long-term survival in patients who underwent AVR $(75.9 \pm 1.6 \%$ vs $82.6 \pm 1.2 \%$ at 6 years, $\mathrm{p}<0.001$, figure 1), whereas no significant difference was observed in patients with MVR or MVRp $(84.4 \pm 2.4 \%$ vs $87.8 \pm 2.0 \%, \mathrm{p}=0.40$ ).

In the multivariable analysis (table 2), POAF remained a significant predictor of long-term mortality in patients with AVR (HR=1.22, CI 1.02 to $1.45, \mathrm{p}=0.03)$. POAF was not significantly associated with survival in patients with MVR or MVRp ( $\mathrm{HR}=0.87$, CI 0.58 to $1.29, \mathrm{p}=0.48)$.

\section{DISCUSSION}

The major finding of this study is that POAF following valvular surgery is associated with long-term mortality in patients with AVR but not in those with MVR or MVRp.
Impact of POAF on long-term survival in patients with valvular surgery

Mariscalco and Engstrom ${ }^{6}$ found no significant association between POAF and long-term survival following valvular surgery, whereas Filardo et $a l^{9}$ reported that POAF after AVR is significantly associated with long-term mortality independently of the preoperative disease severity. Our results are highly consistent with both studies. Indeed, the association between POAF and survival was only borderline in the whole cohort, largely driven by the significant association with survival in patients with AVR. In patients who underwent mitralvalve surgery, we did not find a significant impact of POAF on long-term survival. Our results thus suggest that the lack of association between POAF and survival reported in the study of Mariscalco and Engstrom might be due to the inclusion of a mixed group of patients who underwent AVR and/or mitral surgery. Furthermore, our study has more statistical power than the two reports previously published. The association reported in the study of Mariscalco and Engstrom ( $\mathrm{HR}=1.21$, CI 0.92 to 1.58) which included 995 patients with isolated valvular surgery would probably have been statistically significant in a sample of patients as large as ours.
Figure 1 Long-term survival in patients with or without postoperative atrial fibrillation (POAF) in the whole cohort and in subsets of patients defined according to the type of valvular procedure.
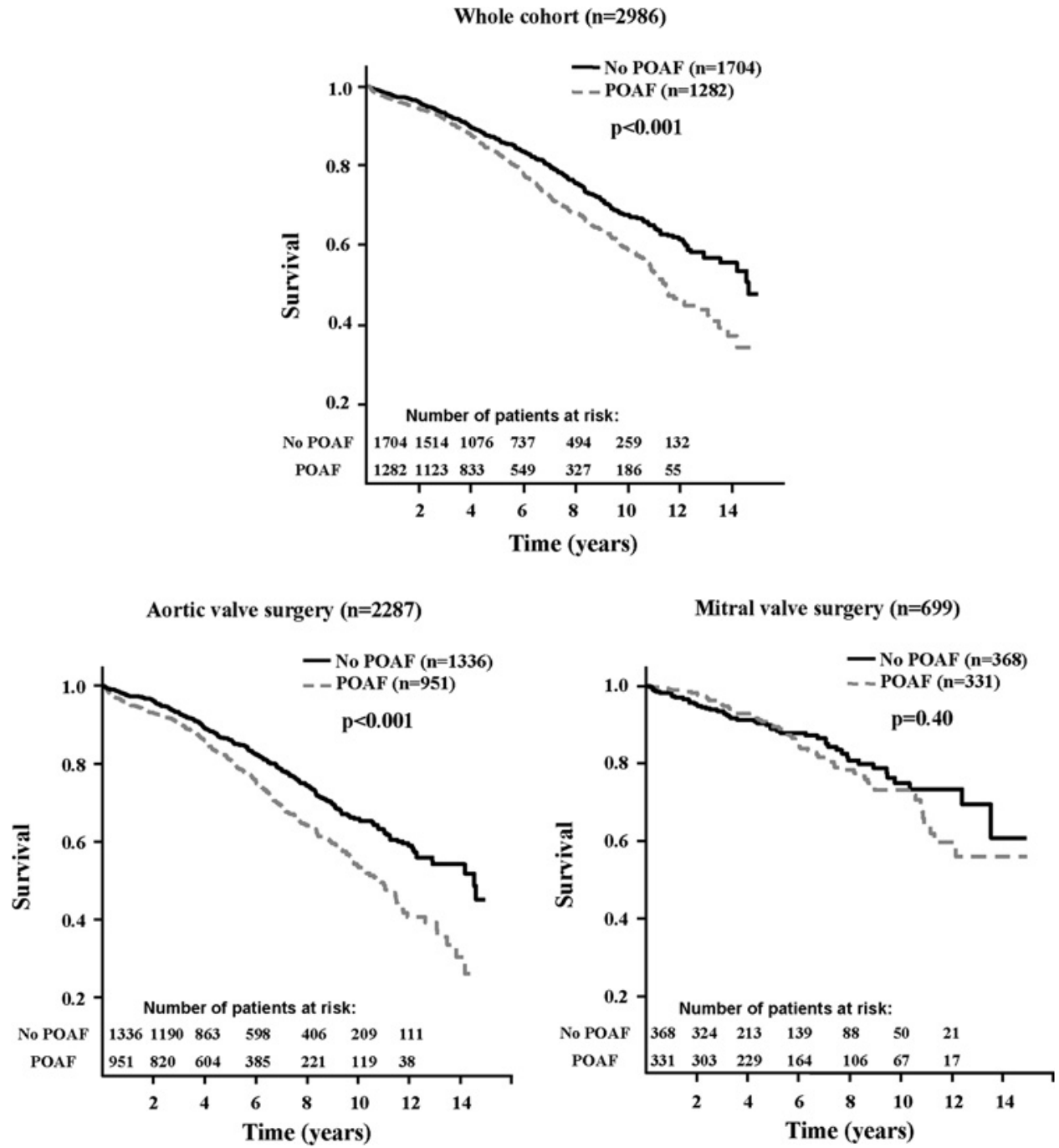
Table 2 Individual and multivariable analysis of the impact of postoperative atrial fibrillation on survival in the whole cohort and in patients with aortic or mitral-valve surgery

\begin{tabular}{|c|c|c|c|c|c|c|}
\hline & \multicolumn{2}{|c|}{ Whole cohort, $\mathrm{n}=2986$} & \multicolumn{2}{|c|}{$\begin{array}{l}\text { Patients undergoing aortic- } \\
\text { valve replacement, } n=2287 \\
\end{array}$} & \multicolumn{2}{|c|}{$\begin{array}{l}\text { Patients undergoing mitral- } \\
\text { valve surgery, } n=699\end{array}$} \\
\hline & HR (95\% Cl) & p Value & HR $(95 \%$ Cl) & p Value & HR $(95 \%$ Cl) & p Value \\
\hline Individual analysis & 1.41 (1.21 to 1.63$)$ & $<0.001$ & $1.53(1.30$ to 1.80$)$ & $<0.001$ & $1.17(0.81$ to 1.68$)$ & 0.40 \\
\hline $\begin{array}{l}\text { Model 1: adjusted on age, } \\
\text { gender and the type of } \\
\text { surgical procedure }\end{array}$ & 1.18 (1.01 to 1.37$)$ & 0.04 & $1.23(1.05$ to 1.46$)$ & 0.01 & $0.86(0.59$ to 1.25$)$ & 0.43 \\
\hline $\begin{array}{l}\text { Model 2: adjusted on age, } \\
\text { gender, comorbidities and } \\
\text { the type of surgical procedure* }\end{array}$ & $1.17(1.00$ to 1.38$)$ & 0.05 & $1.22(1.02$ to 1.45$)$ & 0.03 & 0.87 (0.58 to 1.29$)$ & 0.48 \\
\hline
\end{tabular}

It remains unclear why the significance of the association between POAF and survival differs markedly according to the type of valvular procedure. In our population, patients with AVR were mainly patients with severe aortic stenosis. As aortic stenosis shares some physiopathology similarities with CAD, the association of POAF with long-term survival in patients with AVR appears consistent with the significant association between POAF and long-term survival after isolated CABG. $^{4-8}$ The lack of significant association between POAF and long-term survival after MVR or MVRp may be explained, at least in part, by differences in the factors and mechanisms involved in the pathogenesis of POAF in patients with mitral surgery versus patients with AVR and/or CABG. The determinants of POAF in patients with mitral-valve surgery are largely unknown. To the best of our knowledge, there is no specific study focusing on the prediction of the risk for POAF after mitral-valve surgery. On the other hand, inflammation appears to be the key determinant of POAF following CABG. ${ }^{11-14}$ Consistently, pharmacological interventions targeting postoperative inflammation such as glucocorticoids have been shown to reduce the incidence of POAF after CABG. ${ }^{15}$ Hence, POAF after isolated CABG and AVR could be a marker of exaggerated inflammatory response to stress, which would in turn translate into a higher incidence of perioperative morbidity and longterm mortality. In contrast, POAF after mitral-valve surgery might be linked to the preoperative features of mitral valve disease (left atrial dilation and hypertension, etc), which are, at least in part, corrected by surgery. This hypothesis has to be tested in future studies. In particular, mechanistic studies specifically focusing on the pathogenesis of POAF after aortic and mitral-valve surgery are needed.

\section{Lack of association even in patients without long-term indication for warfarin}

It has been previously hypothesised that POAF might have a weaker effect after valvular surgery because of the indication of lifelong warfarin therapy after mechanical-valve implantation. ${ }^{6}$ In line with this hypothesis, the long-term anticoagulation instituted at the time of mechanicalvalve AVR would prevent embolism related to atrial fibrillation recurrence, thus reducing the impact of POAF on survival. The results of a large observational study recently supported this hypothesis. ${ }^{8}$ In this study, anticoagulant treatment at discharge was associated with decreased long-term mortality in patients who experience POAF after isolated CABG. However, in our cohort, there was no significant interaction between the association of POAF with survival and an indication for longterm warfarin treatment (ie, patients with mechanical valves). This lack of modifying effect of lifetime warfarin treatment would argue against the hypothesis that the lack of impact of POAF after mitral valvular surgery is due to the anticoagulation needed for mechanical valves.

\section{Clinical implications}

According to our results, POAF is a significant predictor of long-term survival after isolated or combined AVR. Consequently, patients with POAF after AVR should be considered at increased risk for longer-term events. Hence, additional specific intervention, possibly a closer follow-up, should be considered in these patients.

\section{Limitations}

This is a large retrospective monocentre observational study. As in any observational study, causality between POAF after AVR and mortality cannot be ascertained. POAF might be a marker of underlying myocardial disease, or other conditions, which are associated with poorer outcome. Besides, the mechanisms involved in the poorer survival of patients with POAF remain uncertain. Postdischarge atrial fibrillation recurrence could be implicated in this excess of mortality. We unfortunately do not have the data regarding postdischarge arrhythmia recurrences. Because of these limitations, the mechanisms by which mortality is explained by postoperative AF remain speculative, and 
the causative link between POAF and survival is still uncertain.

We had no precise information regarding the postdischarge management of POAF. Medications introduced after the episode of POAF may have influenced our analysis. However, as a general rule, only recurrent or sustained episodes of AF after the postoperative period are treated by long-term anticoagulant therapy or antiarrhythmic drugs. Considering the relatively low proportion of patients with persistent AF after the early postoperative period, this residual confounding probably has a minimal impact on the results.

Constant ECG monitoring was performed only during the first $48 \mathrm{~h}$ after surgery. Asymptomatic episodes or transient episodes of POAF after the first $48 \mathrm{~h}$ after surgery might consequently have been underdiagnosed. This misclassification might have biased the results. Besides, no conclusions can be drawn from our results regarding the significance of asymptomatic POAF.

\section{Conclusion}

This study demonstrates that POAF is associated with long-term mortality in patients with AVR but not in those with mitral-valve surgery. The underlying factors involved in the pathogenesis of POAF after mitral-valve surgery may account for this lack of impact of POAF on survival in these patients.

Correction notice The "To cite: ..." information and running footer in this article have been updated with the correct volume number (volume 1).

Funding This work was supported by the Quebec Heart and Lung Institute Foundation.

Competing interests None.

Ethics approval Ethics approval was provided by the Institutional Review Board of Quebec Heart \& Lung Institute.

Contributors NG performed the statistical analyses and drafted the manuscript; JM participated in the interpretation of the data and critically reviewed the manuscript; PP, PV and FD critically reviewed the manuscript and participated in the interpretation of the data; PM participated in the design of the study, interpretation of the data and critical review of the manuscript.

Provenance and peer review Not commissioned; externally peer reviewed.
Data sharing statement No additional data available.

\section{REFERENCES}

1. Echahidi N, Pibarot P, O'Hara G, et al. Mechanisms, prevention, and treatment of atrial fibrillation after cardiac surgery. J Am Coll Cardiol 2008;51:793-801.

2. Lauer MS, Eagle KA, Buckley MJ, et al. Atrial fibrillation following coronary artery bypass surgery. Prog Cardiovasc Dis 1989;31:367-78.

3. Almassi GH, Schowalter T, Nicolosi AC, et al. Atrial fibrillation after cardiac surgery: a major morbid event? Ann Surg 1997;226:501-11; discussion 511-13.

4. Villareal RP, Hariharan R, Liu BC, et al. Postoperative atrial fibrillation and mortality after coronary artery bypass surgery. J Am Coll Cardiol 2004;43:742-8.

5. Filardo G, Hamilton C, Hebeler RF Jr, et al. New-onset postoperative atrial fibrillation after isolated coronary artery bypass graft surgery and long-term survival. Circ Cardiovasc Qual Outcomes 2009;2:164-9.

6. Mariscalco G, Engstrom KG. Postoperative atrial fibrillation is associated with late mortality after coronary surgery, but not after valvular surgery. Ann Thorac Surg 2009;88:1871-6.

7. Mariscalco G, Klersy C, Zanobini M, et al. Atrial fibrillation after isolated coronary surgery affects late survival. Circulation 2008;118:1612-18.

8. El-Chami MF, Kilgo P, Thourani $\mathrm{V}$, et al. New-onset atrial fibrillation predicts long-term mortality after coronary artery bypass graft. $J A m$ Coll Cardiol 2010;55:1370-6.

9. Filardo G, Hamilton C, Hamman B, et al. New-onset postoperative atrial fibrillation and long-term survival after aortic valve replacement surgery. Ann Thorac Surg 2010;90:474-9.

10. Echahidi N, Mohty $\mathrm{D}$, Pibarot $\mathrm{P}$, et al. Obesity and metabolic syndrome are independent risk factors for atrial fibrillation after coronary artery bypass graft surgery. Circulation 2007;116(11 Suppl): I213-19.

11. Girerd N, Pibarot P, Fournier D, et al. Middle-aged men with increased waist circumference and elevated C-reactive protein level are at higher risk for postoperative atrial fibrillation following coronary artery bypass grafting surgery. Eur Heart $J$ 2009;30:1270-8.

12. Ucar HI, Tok M, Atalar E, et al. Predictive significance of plasma levels of interleukin-6 and high-sensitivity C-reactive protein in atrial fibrillation after coronary artery bypass surgery. Heart Surg Forum 2007; 10:E131-5.

13. Bruins $\mathrm{P}$, te Velthuis $\mathrm{H}$, Yazdanbakhsh AP, et al. Activation of the complement system during and after cardiopulmonary bypass surgery: postsurgery activation involves C-reactive protein and is associated with postoperative arrhythmia. Circulation 1997;96:3542-8.

14. Abdelhadi RH, Gurm HS, Van Wagoner DR, et al. Relation of an exaggerated rise in white blood cells after coronary bypass or cardiac valve surgery to development of atrial fibrillation postoperatively. $A m$ J Cardiol 2004;93:1176-8.

15. Halonen J, Halonen $\mathrm{P}$, Jarvinen $\mathrm{O}$, et al. Corticosteroids for the prevention of atrial fibrillation after cardiac surgery: a randomized controlled trial. JAMA 2007;297:1562-7. 